\title{
On Recursively Directed Hypercubes
}

\author{
Carmel Domshlak \\ Department of Computer Science, \\ Ben-Gurion University, Beer-Sheva, Israel \\ dcarmel@cs.bgu.ac.il
}

Submitted: October 23, 2001; Accepted: May 7, 2001.

MR Subject Classifications: 05C62, 05C12, 05C38, 91B08

\begin{abstract}
In this paper we introduce the recursively directed hypercubes, and analyze some of their structural properties. We show that every recursively directed hypercube is acyclic, and has a unique pair of source and sink nodes. The main contribution of the paper is an analysis of distances between the nodes in such a graph. We show that the distance from the source node to any other node, and from any node to the sink node is bounded by $n+1$, where $n$ is the dimension of the hypercube, but the diameter of a recursively directed hypercube may be exponential in $n$.
\end{abstract}

\section{Introduction}

An $n$-dimensional hypercube $H_{n}$, or a Hamming cube, is a graph with $2^{n}$ nodes each labeled by an $n$-bit binary number. Edges occur between nodes whose labels differ in precisely one bit. Recursively, hypercubes can be defined as follows: A 1-dimensional hypercube is an edge with one node labeled 0 and the other node labeled 1 . An $(n+1)$ dimensional hypercube is constructed from two $n$-dimensional hypercubes, $H_{n}^{0}$ and $H_{n}^{1}$, by adding edges from each node in $H_{n}^{0}$ to the node in $H_{n}^{1}$ that has the same label and then by prefixing all of the labels in $H_{n}^{0}$ with a 0 and all of the labels in $H_{n}^{1}$ with a 1.

A directed $n$-dimensional hypercube $\vec{H}_{n}$, as discussed in $[7,12]$, is obtained by an arbitrary orientation of the undirected hypercube $H_{n}$. The motivation for investigating structural properties of the directed hypercubes is given in [1] with respect to the configuration graph of a Hopfield Net [10]. In [7, 12] it is shown that acyclic directed hypercubes may have exponential diameter. More precisely, it was proved that for every $n \geq 1$ there is an acyclic oriented hypercube $\vec{H}_{n}$ with diameter $\geq F_{n+1}$, where $F_{n}$ is the $n$th Fibonacci number.

In turn, design of special-purpose orientations of graphs [4] in general, and of the hypercubes in particular $[3,8,9,11]$, appear in literature as well. These orientations were developed, mainly in the context of computer networks, to offer some important 

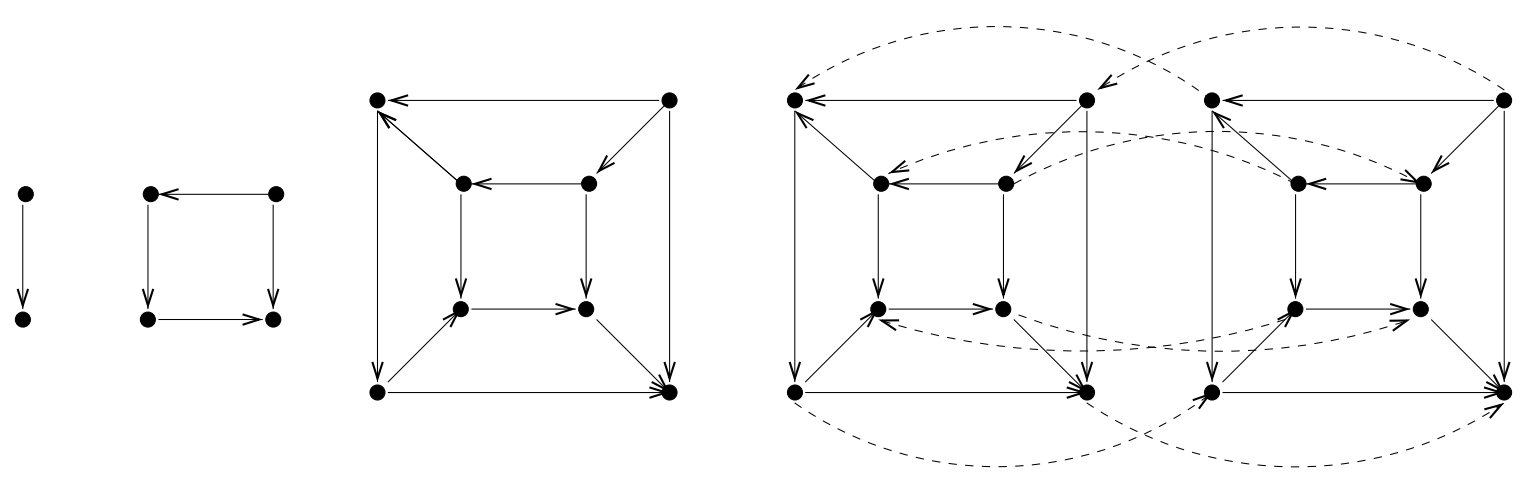

Figure 1: Recursive construction of a directed hypercube.

properties, e.g. high connectivity [3, 9], small diameter [11], both these properties [8], etc. In this paper we take a somewhat opposite direction - we introduce a family of directed hypercubes, and analyze its structural properties.

Observe, that the two components of $H_{n}, H_{n-1}^{0}$ and $H_{n-1}^{1}$, are trivially identical, while, in most of the cases, an oriented $\vec{H}_{n}$ cannot be disassembled into two identically oriented components $\vec{H}_{n-1}^{0}$ and $\vec{H}_{n-1}^{1}$. Now, lets define the directed $n$-dimensional hypercube differently, such that its definition will, in some sense, correlate to the recursive definition of its undirected predecessor:

1. A 1-dimensional directed hypercube is an edge with one node labeled 0 and the other node labeled 1.

2. An $(n+1)$-dimensional directed hypercube is constructed from two identical $n$-dimensional directed hypercubes, $\vec{H}_{n}^{0}$ and $\vec{H}_{n}^{1}$, by adding arbitrarily directed edges between each node in $\vec{H}_{n}^{0}$ and the node in $\vec{H}_{n}^{1}$ that has the same label and then by prefixing all of the labels in $\vec{H}_{n}^{0}$ with a 0 and all of the labels in $\vec{H}_{n}^{1}$ with a 1 .

In what follows, a pair of identically labeled nodes in $\vec{H}_{n}^{0}$ and $\vec{H}_{n}^{1}$ are called twin nodes.

An example of such a recursive construction is presented in Figure 1. As far as we know, such a definition of the directed $n$-dimensional hypercube is novel, and thus the properties of this structure were not previously investigated. In order to distinguish between the two definitions of the directed hypercube in what follows, we denote such a structure by recursively directed hypercube.

In Artificial Intelligence, recursively directed hypercubes represent a preference order over the outcomes, defined by a Conditional Preference Network (CP-net) $[2,6]$. As such, the diameter of the hypercube gives a lower bound on any algorithm for preferential dominance testing for a pair of outcomes, therefore, we are motivated to consider the structural properties of such directed hypercubes, and especially their diameter.

In this paper, we first show that each recursively directed hypercube is acyclic and have a unique pair of source and sink nodes. Second, we show that the distances from the source node to any other node, and from any node to the sink node in any $\vec{H}_{n}$ are 
bounded by $n$. However, in spite of such a strong property, we show that recursively directed hypercube may have exponential diameter.

The rest of the paper is organized as follows: Section 2 presents some general properties of the recursively directed hypercubes, e.g. its acyclicity. Section 3 is devoted to an analysis of the distance between the nodes in recursively directed hypercubes. Section 4 provides an additional way to define recursively directed hypercubes, which allows us to pose an open question. In Section 5 we conclude.

\section{General Properties}

In this section we show that any recursively directed hypercube is acyclic, and that it has a unique pair of source and sink nodes. Denote the nodes of $\vec{H}_{n}^{0}$ and $\vec{H}_{n}^{1}$ by $v^{1}, \ldots, v^{2^{n}}$ and $v^{1^{\prime}}, \ldots, v^{2^{n \prime}}$ respectively. In what follows, we use a subscript index for the nodes, e.g. $v_{i}$, in order to specify nodes not according to their actual labels.

Theorem 1 Every recursively directed hypercube is acyclic.

Proof: The proof is by induction on the dimension of the hypercube. Obviously, any $\vec{H}_{1}$ is acyclic since it consists of only one edge. Under the assumption that any $\vec{H}_{n}$ is acyclic we prove that any $\vec{H}_{n+1}$ is acyclic.

Assume to the contrary that there exist some recursively directed hypercube $\vec{H}_{n+1}$ that is not acyclic, and consider an arbitrary cycle in it. Since both $\vec{H}_{n}^{0}$ and $\vec{H}_{n}^{1}$ are acyclic, this cycle contains some edges $e_{1}, \ldots, e_{m}$ (in this order) connecting pairs of twin nodes in $\vec{H}_{n}^{0}$ and $\vec{H}_{n}^{1}$, where $m=2 k$, for some $k \in \mathbb{N}$. Let $e_{1}$ be an edge connecting a node in $\vec{H}_{n}^{0}$ with its twin in $\vec{H}_{n}^{1}$. Then, for $1 \leq i \leq m$, if $i$ is odd then $e_{i}=\left(v_{i}, v_{i}^{\prime}\right)$, otherwise $e_{i}=\left(v_{i}^{\prime}, v_{i}\right)$. Now, for $1 \leq i \leq \frac{m}{2}-1$, there is a path from $v_{(2 i-1)}{ }^{\prime}$ to $v_{(2 i)}{ }^{\prime}$ in $\vec{H}_{n}^{1}$, and a path from $v_{(2 i)}$ to $v_{(2 i+1)}$ in $\vec{H}_{n}^{0}$. Likewise, there is a path from $v_{m}$ to $v_{1}$ in $\vec{H}_{n}^{0}$. However, $\vec{H}_{n}^{0}$ and $\vec{H}_{n}^{1}$ are identical, thus, for $1 \leq i \leq \frac{m}{2}-1$, there is a path from $v_{(2 i-1)}$ to $v_{(2 i)}$ in $\vec{H}_{n}^{0}$, which contradicts our assumption that $\vec{H}_{n}^{0}$ is acyclic.

Now, consider a directed hypercube $\vec{H}_{n}$, and a node $v \in \vec{H}_{n}$. The node $v$ is a source of $\vec{H}_{n}$ if there is no edge $\left(v^{\prime}, v\right) \in \vec{H}_{n}$. Similarly, the node $v$ is a sink of $\vec{H}_{n}$ if there is no edge $\left(v, v^{\prime}\right) \in \vec{H}_{n}$.

Note that, in general, there is no particular limitations on the number of the sources and/or the sinks for directed hypercubes. For example, the directed hypercube in Figure 2(a) has two sources and two sinks, while the directed hypercube in Figure 2(b) has neither sources, nor sinks. The only observation is that any acyclic directed hypercube should contain at least one source and one sink.

Theorem 2 Every recursively directed hypercube has exactly one source and one sink.

Proof: The proof is by induction on the dimension of the hypercube. Obviously, any $\vec{H}_{1}$ has a unique source and a unique sink since it consists of only one edge. Under the assumption that any $\vec{H}_{n}$ has a unique source and a unique sink, we prove it for any $\vec{H}_{n+1}$. 


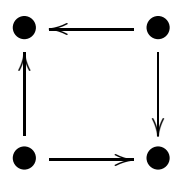

(a)

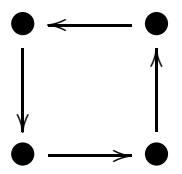

(b)

Figure 2: Examples of general directed hypercubes.

Consider a recursively directed hypercube $\vec{H}_{n+1}$, and its identical components $\vec{H}_{n}^{0}$ and $\vec{H}_{n}^{1}$. Denote the sources and sinks of $\vec{H}_{n}^{0}$ and $\vec{H}_{n}^{1}$ by $\{s, t\}$ and $\left\{s^{\prime}, t^{\prime}\right\}$, respectively. The only candidates for sources of $\vec{H}_{n+1}$ are the twin nodes $s$ and $s^{\prime}$. However, by definition of the directed hypercube, either $s$ has an incoming edge $\left(s^{\prime}, s\right)$ or $s^{\prime}$ has an incoming edge $\left(s, s^{\prime}\right)$, thus exactly one of them becomes the source of $\vec{H}_{n+1}$. Hence, we proved that $\vec{H}_{n+1}$ has exactly one source. Similarly we can show that only one of the twin nodes $t$ and $t^{\prime}$ is the sink of $\vec{H}_{n+1}$.

\section{Diameter of Recursively Directed Hypercubes}

In this section we analyze distances between the nodes in a recursively directed hypercube. We show that the distance from the source node to any other node, and the distance from any node to the sink node is less or equal to $n+1$, where $n$ is the degree of the hypercube. However, we show that the distance between two arbitrary nodes in a recursively directed hypercube may be exponential in its degree.

We measure the length of a path in a directed graph by the number of nodes on the path, and we denote the length of the shortest path from a node $v$ to a node $u$ by $l(v, u)$. If there is no path from $v$ to $u$, then we define $l(v, u)=0$. Observe that if there is a path from $v$ to $u$, then $l(v, u)>0$.

Note that the customary choice for the case that $u$ is not reachable from $v$, is to define $l(v, u)=\infty[5]$. In our case, such a decision will affect the simplicity of some of the forthcoming definitions, e.g. Eq. 1 and Eq. 2. From the same reasons, in [7], where the length of a path was measured by the number of edges on the path, $l(v, u)$ for the case that $u$ is not reachable from $v$ was defined to be -1 .

Theorem 3 Let $s$ and $t$ be the source and the sink nodes of a recursively directed hypercube $\vec{H}_{n}$. Then, for any node $v$ in $\vec{H}_{n}, 1 \leq l(s, v) \leq n+1$ and $1 \leq l(v, t) \leq n+1$.

Proof: An immediate implication of theorem 2 is that any node in a recursively directed hypercube is accessible from the source node, and that the sink node is accessible from any node. Therefore, for any node $v \in \vec{H}_{n}$, both $l(s, v) \geq 1$ and $l(v, t) \geq 1$. The proof of $l(s, v) \leq n+1$ is by induction on the the dimension of the hypercube. In any $\vec{H}_{1}$ there is only one edge, thus the length of any path in $\vec{H}_{1}$ cannot exceed 2. Under the assumption that the claim is true for any $\vec{H}_{n}$, we prove that it remains true for any $\vec{H}_{n+1}$. 
The proof is simple: Consider the source node $s$ and any other node $v$ of $\vec{H}_{n+1}$. Without loss of generality, suppose that $s \in \vec{H}_{n}^{0}$, where $\vec{H}_{n}^{0}$ is one of the two recursive components of $\vec{H}_{n+1}$. If $v \in \vec{H}_{n}^{0}$, then, from the induction hypothesis, $l(s, v) \leq n+1$, since $s$ is also the source node of $\vec{H}_{n}^{0}$. Alternatively, if $v \in \vec{H}_{n}^{1}$, consider the twin node $s^{\prime}$ of $s$, which is also the source node of $\vec{H}_{n}^{1}$. From the induction hypothesis, $l\left(s^{\prime}, v\right) \leq n+1$. Now, since the edge $\left(s, s^{\prime}\right)$ belongs to $\vec{H}_{n+1}$, we proved that $l(s, v) \leq n+2$. Similarly we can show that $l(v, t) \leq n+1$.

Note that theorem 3 trivially implies that the length of a shortest path from $s$ to $t$ via any specified node $x$ is bounded by $2 n+1$. However, the following theorem shows that, in spite of such a nice property, recursively directed hypercube may have exponential diameter.

Theorem 4 For every $n \geq 1$ there is a recursively directed hypercube $\vec{H}_{n}$ with diameter

$$
d\left(\vec{H}_{n}\right) \geq\left[\frac{2}{3} \sum_{j=1}^{\lfloor 0.63 n\rfloor-1} a_{j}\right]+a_{\lfloor 0.63 n\rfloor-1}-n
$$

where $\left\{a_{0}=2 ; \quad a_{m}=\left\lfloor\frac{3}{2} a_{m-1}\right\rfloor, m \geq 1\right\}$.

The proof of theorem 4 is constructive and is based on the two following lemmata. Informally, the construction of such $\vec{H}_{n}$ in performed in two subsequent, differently defined, recursive processes. First, a recursively directed hypercube of a particular dimension $i$ is created from $\vec{H}_{1}$ according to the construction from the proof of lemma 1. Second, a recursively directed hypercube $\vec{H}_{n}$ is created from $\vec{H}_{i}$ according to the construction from the proof of lemma 2 . The obtained $\vec{H}_{n}$ is then shown to have the diameter with the above lower bound.

Lemma 1 For every $n \geq 1$ there is a recursively directed hypercube $\vec{H}_{n}$ with a path through its all $2^{n}$ nodes.

Proof: The proof is by inductive construction of such an hypercube. In any $\vec{H}_{1}$, its unique edge represents the path of length 2 . Under the assumption that there exist a recursively directed hypercube $\vec{H}_{n}$ that contains a path through its $2^{n}$ nodes, we construct a recursively directed hypercube $\vec{H}_{n+1}$ that contains a path of length $2^{n+1}$.

For the simplicity of notation, we number the nodes of the corresponding $\vec{H}_{n}$ according to their appearance on the path of length $2^{n}$, and not according to their actual labels. First, the $(n-1)$-dimensional components of the required $\vec{H}_{n+1}$ are created by duplicating $\vec{H}_{n}$ into $\vec{H}_{n}^{0}$ and $\vec{H}_{n}^{1}$. Then, the twin nodes of $\vec{H}_{n}^{0}$ and $\vec{H}_{n}^{1}$ are connected as follows. For $1 \leq i \leq 2^{n}$, if $i$ is odd then $\left(v_{i}, v_{i}^{\prime}\right) \in \vec{H}_{n+1}$, otherwise $\left(v_{i}^{\prime}, v_{i}\right) \in \vec{H}_{n+1}$. Clearly, the constructed $\vec{H}_{n+1}$ contains the path:

$$
1 \cdot 1^{\prime} \cdot 2^{\prime} \cdot 2 \cdot 3 \cdot 3^{\prime} \cdot \ldots \cdot(2 j-1) \cdot(2 j-1)^{\prime} \cdot(2 j)^{\prime} \cdot(2 j) \cdot(2 j+1) \cdot(2 j+1)^{\prime} \ldots \cdot\left(2^{n}-1\right)^{\prime} \cdot\left(2^{n}\right)^{\prime} \cdot\left(2^{n}\right)
$$

which is of length $2^{n+1}$. 
The diameter of $\vec{H}_{n}=\left(V_{n}, E_{n}\right)$, denoted by $d\left(\vec{H}_{n}\right)$, is given by

$$
d\left(\vec{H}_{n}\right)=\max \left\{l\left(v_{i}, v_{j}\right) \mid v_{i}, v_{j} \in V_{n}\right\}
$$

Let us extend the notion of diameter as follows. The $k$-nodes constrained diameter of $\vec{H}_{n}$, denoted by $\delta\left(\vec{H}_{n}, k\right)$ is given by

$$
\delta\left(\vec{H}_{n}, k\right)=\max \left\{\sum_{i=1}^{k-1} l\left(v_{i}, v_{i+1}\right) \mid\left\{v_{1}, \ldots, v_{k}\right\} \subseteq V_{n}\right\}
$$

In particular, $\delta\left(\vec{H}_{n}, 2\right)=d\left(\vec{H}_{n}\right)$. The actual $k$ nodes defining a $k$-nodes restricted path $\rho$ are called the skeleton of $\rho$. We make a convention that if there is no path in $\vec{H}_{n}$ through a set of $k$ nodes then $\delta\left(\vec{H}_{n}, k\right)=0$.

Lemma 2 Given a recursively directed hypercube $\vec{H}_{n}$ with $\delta\left(\vec{H}_{n}, k\right)>0$, we can create a recursively directed hypercube $\vec{H}_{n+1}$ with

$$
\delta\left(\vec{H}_{n+1},\left\lceil\frac{2}{3} k\right\rceil\right) \geq \delta\left(\vec{H}_{n}, k\right)+\left\lceil\frac{2}{3} k\right\rceil-1
$$

Proof: Suppose that $\delta\left(\vec{H}_{n}, k\right)$ is obtained on a path $\rho_{n}$ with a skeleton $\left\{v_{1}, \ldots, v_{k}\right\} \subseteq V_{n}$. In what follows, by the set of nodes between the nodes $v$ and $u$ we refer to the nodes that are reachable from $v$ and that $u$ is reachable from them (trivially, if this set is not empty then it contains the nodes $v$ and $u$ ).

Lets assume that $k=3 j$ for some $j \in \mathbb{N}$. The construction of the required hypercube $\vec{H}_{n+1}$ from the duplicates $\vec{H}_{n}^{0}$ and $\vec{H}_{n}^{1}$ of $\vec{H}_{n}$ is as follows. For each node $v_{i}$ from the skeleton of $\rho_{n}$, such that $i=3 m-1$ for some $m \in \mathbb{N}$, we add to $\vec{H}_{n+1}$ the edge $\left(v_{i}, v_{i}^{\prime}\right)$. For each remaining node $x$ in $\vec{H}_{n}$ we add to $\vec{H}_{n+1}$ the edge $\left(x^{\prime}, x\right)$. Informally, the former edges are the only "bridges" from $\vec{H}_{n}^{0}$ to $\vec{H}_{n}^{1}$.

Now consider the shortest path $\rho_{n+1}$ in $\vec{H}_{n+1}$ from $v_{1}$ to $v_{k}^{\prime}$ via the following nodes:

$$
v_{1}, v_{3}{ }^{\prime}, v_{4}, v_{6}{ }^{\prime}, v_{7}, \ldots, v_{3 i-2}, v_{3 i}^{\prime}, v_{3(i+1)-2}, \ldots, v_{k}^{\prime}, \quad 1 \leq i \leq j
$$

Informally, the skeleton of this path is obtained from the skeleton of $\rho_{n}$ by (i) removing all the nodes $v_{3 i-1}$; (ii) using the nodes $v_{3 i-2}$ from $\rho_{n}$ in $\vec{H}_{n}^{0}$; (iii) using the twin nodes $v_{3 i}^{\prime}$ in $\vec{H}_{n}^{1}$, for $1 \leq i \leq j$. Such a construction of $\vec{H}_{n+1}$ is illustrated in Figure $3($ a), while the corresponding $\rho_{n+1}$ is presented in Figure 3(b). In this figure, the thin arrows stand for paths, while the thick arrows (both solid and dashed) stands for edges.

First, observe that for $1 \leq i \leq j$,

$$
l\left(v_{3 i}{ }^{\prime}, v_{3(i+1)-2}, \vec{H}_{n+1}\right)=l\left(v_{3 i}, v_{3(i+1)-2}, \vec{H}_{n}\right)+1
$$

since we can use the edge $\left(v_{3 i}{ }^{\prime}, v_{3 i}\right)$ in order to move from $\vec{H}_{n}^{1}$ to $\vec{H}_{n}^{0}$, and then to use the shortest path from $v_{3 i}$ to $v_{3(i+1)-2}\left(v_{3(i+1)-2} \equiv v_{3 i+1}\right)$. On the other hand, for $1 \leq i \leq j$,

$$
l\left(v_{3 i-2}, v_{3 i}{ }^{\prime}, \vec{H}_{n+1}\right)=l\left(v_{3 i-2}, v_{3 i-1}, \vec{H}_{n}\right)+l\left(v_{3 i-1}, v_{3 i}, \vec{H}_{n}\right)+1
$$




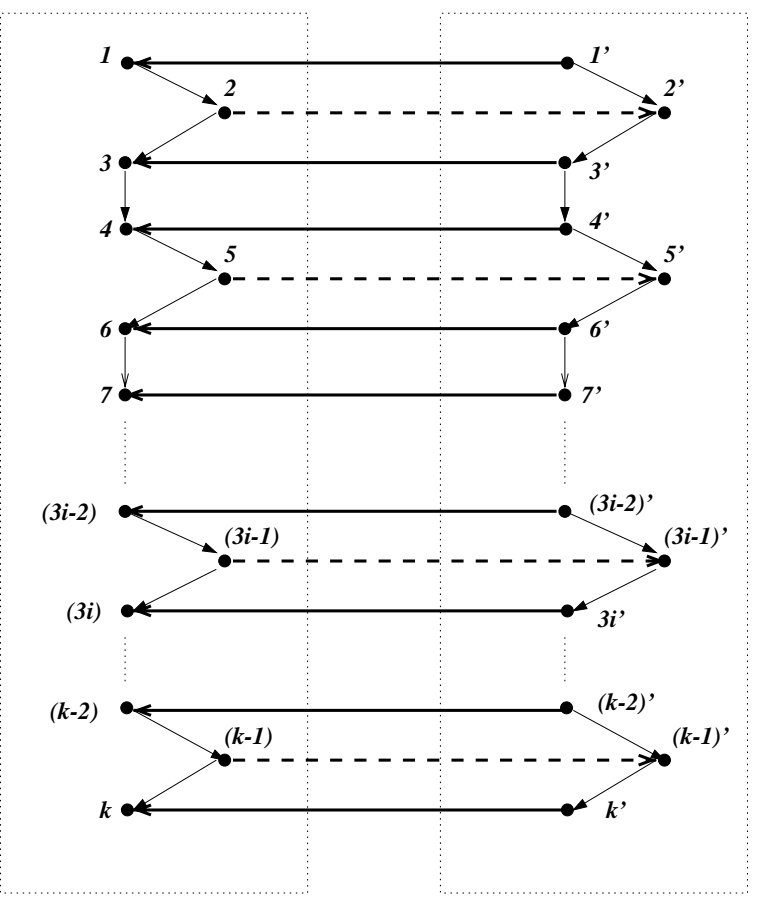

(a)

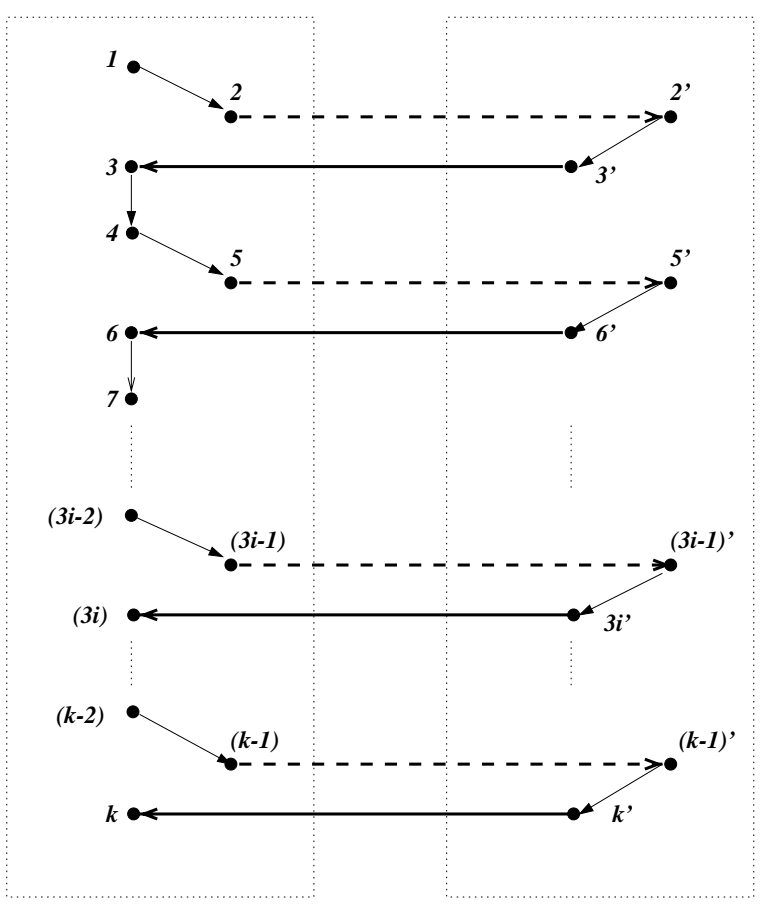

(b)

Figure 3: (a) $\vec{H}_{n+1}$; (b) $\rho_{n+1}$.

since $v_{3 i-1}$ is the only bridge from $\vec{H}_{n}^{0}$ to $\vec{H}_{n}^{1}$ between $v_{3 i-2}$ and $v_{3 i}$. Thus, first we take the shortest path in $\vec{H}_{n}^{0}$ from $v_{3 i-2}$ to $v_{3 i-1}$, then we use the edge $\left(v_{3 i-1}, v_{3 i-1}^{\prime}\right)$, and then we take the shortest path in $\vec{H}_{n}^{1}$ from $v_{3 i-1}^{\prime}$ to $v_{3 i}^{\prime}$. Since $k=3 j$, the path $\rho_{n+1}$ is defined by $\frac{2}{3} k$ nodes in $\vec{H}_{n+1}$, and from Eq. 3, and Eq. 4 follows that:

$$
\left|\rho_{n+1}\right|=\left|\rho_{n}\right|+\frac{2}{3} k-1
$$

because there are $\frac{2}{3} k-1$ traversals between $\vec{H}_{n}^{0}$ and $\vec{H}_{n}^{1}$. Recall that $\rho_{n}$ was chosen such that $\left|\rho_{n}\right|=\delta\left(\vec{H}_{n}, k\right)$, thus

$$
\delta\left(\vec{H}_{n+1}, \frac{2}{3} k\right) \geq \delta\left(\vec{H}_{n}, k\right)+\frac{2}{3} k-1
$$

The analysis of the remaining cases of $k=3 j-1$ and $k=3 j-2$ is similar except for the following details. If $k=3 j-1$, then we consider the shortest path $\rho_{n+1}$ in $\vec{H}_{n+1}$ from $v_{1}$ to $v_{k}^{\prime}$ via the nodes:

$$
v_{1}, v_{3}{ }^{\prime}, v_{4}, v_{6}{ }^{\prime}, v_{7}, \ldots, v_{3 i-2}, v_{3 i}^{\prime}, v_{3(i+1)-2} \ldots, v_{k}{ }^{\prime}, \quad 1 \leq i \leq j
$$

The only difference between this $\rho_{n+1}$ and the one for the case of $k=3 j$ is that we remove the nodes $v_{3 i-1}$ from its skeleton, but for $1 \leq i \leq j-1$. For the last node $v_{3 j-1}=v_{k}$ we 
use its representative $v_{k}{ }^{\prime}$ in $\vec{H}_{n}^{1}$. Here the path $\rho_{n+1}$ is defined by $\left\lceil\frac{2}{3} k\right\rceil$ nodes in $\vec{H}_{n+1}$, and thus

$$
\delta\left(\vec{H}_{n+1},\left\lceil\frac{2}{3} k\right\rceil\right) \geq \delta\left(\vec{H}_{n}, k\right)+\left\lceil\frac{2}{3} k\right\rceil-1
$$

Alternatively, if $k=3 j-2$, then we consider the shortest path $\rho_{n+1}$ in $\vec{H}_{n+1}$ from $v_{1}$ to $v_{k}^{\prime}$ via the nodes:

$$
v_{1}, v_{3}{ }^{\prime}, v_{4}, v_{6}{ }^{\prime}, v_{7}, \ldots, v_{3 i-2}, v_{3 i}^{\prime}, v_{3(i+1)-2} \ldots, v_{k}, \quad 1 \leq i \leq j
$$

where the construction of $\rho_{n+1}$ is similar to the one for the case of $k=3 j$. Here the path $\rho_{n+1}$ is also defined by $\left\lceil\frac{2}{3} k\right\rceil$ nodes in $\vec{H}_{n+1}$, and thus

$$
\delta\left(\vec{H}_{n+1},\left\lceil\frac{2}{3} k\right\rceil\right) \geq \delta\left(\vec{H}_{n}, k\right)+\left\lceil\frac{2}{3} k\right\rceil-1
$$

Proof of Theorem 4: Consider the sequence:

$$
a_{0}=2 ; \quad a_{m}=\left\lfloor\frac{3}{2} a_{m-1}\right\rfloor, m \geq 1
$$

From lemma 2 follows that if we can construct a recursively directed hypercube $\vec{H}_{i}$ that contains a path through $a_{n-i}$ nodes, then, using the construction from the proof of lemma 2 recursively (starting from $\vec{H}_{i}$ ), we can construct a recursively directed hypercube $\vec{H}_{n}$ with

$$
\begin{aligned}
d\left(\vec{H}_{n}\right) & \geq a_{n-i}+\sum_{j=1}^{n-i}\left(\left\lceil\frac{2}{3} a_{j}\right\rceil-1\right)=a_{n-i}+\left(\sum_{j=1}^{n-i}\left\lceil\frac{2}{3} a_{j}\right\rceil\right)-(n-i) \\
& \geq a_{n-i}+\left(\frac{2}{3} \sum_{j=1}^{n-i} a_{j}\right)-(n-i)
\end{aligned}
$$

Lemma 1 claims that for any $m \geq 1$ we can create a recursively directed hypercube $\vec{H}_{m}$ with a path through all its $2^{m}$ nodes. Suppose that for a given $n$ we have some $1 \leq i \leq n$ such that $a_{n-i} \leq 2^{i}$. If so, then we can (i) recursively construct $\vec{H}_{i}$ using the construction from the proof of lemma 1 ; (ii) choose a path in $\vec{H}_{i}$ through $a_{n-i}$ nodes, which serve us a basis to the next step; (iii) recursively construct $\vec{H}_{n}$ using the construction from the proof of lemma 1. Note that, by construction, the lower bound for the diameter of the obtained $\vec{H}_{n}$ is presented by Eq. 5 .

Now we want to determine the minimal appropriate $i$ (providing us the maximal appropriate $\left.a_{n-i}\right)$, which will give us the highest lower bound for $d\left(\vec{H}_{n}\right)$. Instead of analyzing the inequality $a_{n-i} \leq 2^{i}$, we analyze the inequality

$$
2 \cdot\left(\frac{3}{2}\right)^{n-i} \leq 2^{i}
$$


Since $2\left(\frac{3}{2}\right)^{j} \geq a_{j}$ for all $j \geq 0$, the minimal $i$ satisfying Eq. 6 will provide an upper bound for the minimal $i$ satisfying $a_{n-i} \leq 2^{i}$. Solving Eq. 6 for $i$ brings us to

$$
i \geq n-(n-1) \log _{3} 2=0.37 n+0.63
$$

thus the required, minimal integer value of $i$ is

$$
i=\lceil 0.37 n+0.63\rceil
$$

From Eq. 5 and Eq. 7 follows that

$$
\begin{aligned}
d\left(\vec{H}_{n}\right) & \geq\left[\frac{2}{3} \sum_{j=1}^{n-\lceil 0.37 n+0.63\rceil} a_{j}\right]+a_{n-\lceil 0.37 n+0.63\rceil}-(n-i) \\
& \geq\left[\frac{2}{3} \sum_{j=1}^{\lfloor 0.63 n\rfloor-1} a_{j}\right]+a_{\lfloor 0.63 n\rfloor-1}-n
\end{aligned}
$$

\section{Alternative View on Recursively Directed Hyper- cubes}

The recursively directed hypercube can be defined in a non-recursive manner. Recall that each node in an $n$-dimensional hypercube is uniquely specified by some assignment on $n$ ordered bits. In what follows, the assignment provided by the label of a node $v$ to the bit $i$ and to the first $i$ bits, are referred to as $v[i]$ and $\left.v\right|_{i}$, respectively.

Proposition 1 A directed hypercube $\vec{H}_{n}$ is recursively directed if and only if there exists an ordering $x_{1}, \ldots, x_{n}$ of the label bits, and a set of boolean orientation functions

$$
\mathcal{F}=\left\{\varphi_{1}(), \varphi_{2}\left(x_{1}\right), \ldots, \varphi_{i}\left(x_{1}, \ldots, x_{i-1}\right), \ldots, \varphi_{n}\left(x_{1}, \ldots, x_{n-1}\right)\right\}
$$

such that, for each edge $(v, u) \in H_{n}$, if the labels of $v$ and $u$ disagree on $x_{i}$ and $v[i]=0$ $(u[i]=1)$, then

$$
\begin{array}{llll}
\varphi_{i}\left(\left.v\right|_{i-1}\right)=0 & \rightarrow & (v, u) \in \vec{H}_{n} \\
\varphi_{i}\left(\left.v\right|_{i-1}\right)=1 & \rightarrow & (u, v) \in \vec{H}_{n}
\end{array}
$$

Note that, $\left.v\right|_{i-1}=\left.u\right|_{i-1}$, thus we can use $\varphi_{i}\left(\left.v\right|_{i-1}\right)$ and $\varphi_{i}\left(\left.u\right|_{i-1}\right)$ interchangeably.

Proposition 1 actually provides an alternative definition for the recursively directed hypercubes. By itself, such a definition is less intuitive than the original one, but it 
provides an additional view on the nature of this structure. Likewise, such a view on the recursively directed hypercubes allows us to pose a following open question, which is important in the scope of the CP-nets.

By definition, for $1 \leq i \leq n$, the arity of the orientation function $\varphi_{i}$ is $i-1$. Intuitively it means that during the construction of the recursively directed hypercube $\vec{H}_{i}$, we may be required to specify explicitly the direction of each one of the edges connecting $\vec{H}_{i-1}^{0}$ and $\vec{H}_{i-1}^{1}$. In turn, if the arity of $\varphi_{i}$ is less than $i-1$, it is possible to specify the direction of the edges connecting $\vec{H}_{i-1}^{0}$ and $\vec{H}_{i-1}^{1}$ in "bulks". If so, what can be said about the diameter of a recursively directed hypercube that is defined by a set of orientation functions with arities bounded by a constant $k$ ?

\section{Summary}

In this paper we presented recursively directed hypercubes, and analyzed their structural properties. We showed that every recursively directed hypercube is acyclic, and has a unique pair of the source and sink nodes. The main contribution of the paper is an analysis of distances between the nodes in such a graph. In particular we showed that the distance from the source node to any other node, and from any node to the sink node is bounded by $n+1$, where $n$ is the dimension of the hypercube, but the diameter of a recursively directed hypercube may be exponential in $n$. Finally, we presented an an open question in respect to a diameter of a subclass of recursively directed hypercubes.

\section{Acknowledgments}

The author wish to thank Michael Lomonosov and Ronen Brafman for many useful discussions, and the referees for their helpful comments.

\section{References}

[1] P. Baldi. Neural networks, acyclic orientations of the hypercube, and sets of orthogonal vectors. SIAM Journal of Discrete Mathematics, 1(1):1-13, 1988.

[2] C. Boutilier, R. Brafman, H. Hoos, and D. Poole. Reasoning with Conditional Ceteris Paribus Preference Statements. In Proceedings of the Fifteenth Annual Conference on Uncertainty in Artificial Intelligence, pages 71-80. Morgan Kaufmann Publishers, 1999.

[3] C. H. Chou and D. Du. Uni-directional Hypercubes. In Proceedings of Third International Conference on Supercomputing, pages 254-263. ACM Digital Library, 1990.

[4] V. Chvátal and C. Thomassen. Distances in Orientations of Graphs. Journal of Combinatorial Theory B, 24(1):61-75, 1978. 
[5] T. H. Cormen, C. E. Leiserson, and R. L. Rivest. Introduction to Algorithms. MIT Press, 1990.

[6] C. Domshlak and R. Brafman. CP-nets - Reasoning and Consistency Testing. In Proceedings of the Eighth International Conference on Principles of Knowledge Representation and Reasoning, pages 121-132, Toulouse, France, April 2002.

[7] H. Everett and A. Gupta. Acyclic directed hypercubes may have exponential diameter. Information Processing Letters, 32(5):243-245, 1989.

[8] P. Fraigniaud, J-C. König, and E. Lazard. Oriented hypercubes. to appear in Networks, 2002.

[9] M. Hamdi. Topological Properties of the Directional Hypercube. Information Processing Letters, 53:277-286, 1995.

[10] J. Hopfield. Neural networks and physical systems with emergent collective computational abilities. Proceedings of the National Academy of Science of USA, 79:25542558, 1982.

[11] J. McCanna. Orientations of the $n$-cube with minimum diameter. Discrete Mathematics, 68:309-310, 1988.

[12] K. Qiu and H. Meijer. A note on diameter of acyclic directed hypercubes. Information Processing Letters, 36(1):51-52, 1990. 